
\title{
28 Research Soure \\ Unhealthy lifestyle is an important risk factor for BPPV recurrence
}

\section{Chang-yong Fu}

Southeast University Zhongda Hospital https://orcid.org/0000-0001-7710-5685

\section{Zhen-zhong Zhang}

Tongde Hospital Of Zhejiang Province

\section{Jin Chen}

Southeast University Zhongda Hospital

\section{Sandip Kumar Jaiswal}

Southeast University Zhongda Hospital

Fu-ling Yan ( $\nabla$ yanfuling218@163.com )

Department of Neurology, affiliated Zhongda Hospital, Southeast University, Nanjing, China

\section{Research article}

Keywords: BPPV, Recurrence, Physical Activity, Recumbent position time

Posted Date: June 2nd, 2020

DOI: https://doi.org/10.21203/rs.3.rs-28421/v1

License: (c) (1) This work is licensed under a Creative Commons Attribution 4.0 International License. Read Full License 


\section{Abstract}

Background: Benign paroxysmal positional vertigo (BPPV) is self-limiting and recurrent but the cost is considerable. The recurrence of BPPV increased significantly under the quarantine policy in Hangzhou. The unhealthy lifestyle risk factors of the recurrence of BPPV have not yet been investigated. So, the objective is to analyze the unhealthy lifestyle risk factors of recurrent BPPV.

Methods: A retrospective observational study was conducted in the Department of Neurology in Tongde Hospital of Zhejiang Province from December 2018 to March 2020. The study included 186 patients aged 23-86 years. All patients received the definitive diagnosis and canalith repositioning maneuvers (CRM) treatment and finally accomplished follow-up for one year. Demographic variables, potential recurrence risk factors, neurological examination, cranial computed tomography and lifestyle were assessed.

Data Analyses: The t-test or chi-squared test was first performed for group comparison, then logistic regression analysis was performed to investigate the recurrence risk factors in all of the patients.

Results: The 1-year recurrence rates of BPPV patients after reposition maneuvers were $36.02 \%$, is significantly higher than other study especially in the first quarter of 2020 (38.8\% of patients with BPPV recurrence). Logistic regression analysis shows that BPPV patients who was poor physical activities and prolonged recumbent position time have more recurrence risks. Poor physical activities (odds ratio $=5.690,95 \%$ confidence interval: $1.650-19.618, p<0.006$ ), prolonged recumbent position time (odds ratio $=1.651,95 \%$ confidence interval: $1.370-2.003, p=0.000)$, were risk factors for the recurrence of BPPV in patients.

Conclusion: We conclude that poor physical activities and prolonged recumbent position time may be independent risk factors for the recurrence of BPPV patients, but aging, Ménière's disease, sudden deafness migraine, hypertension, hyperlipemia, diabetes, $\mathrm{CHD}, \mathrm{POCl}$, mental factors, and mental labor do not increase the recurrence risk.

\section{Background}

Dizziness is a common symptom of benign paroxysmal positional vertigo (BPPV). The cost of evaluating dizziness is considerable. BPPV is the most common peripheral vertigo disease (1), It amounts to $20 \%$ of all Vertigo patients (2). BPPV has a recurrence rate of about $15 \%$ every year (3). However, some studies have found that the highest recurrence rate $(29.67 \%(4))$ is in the age band of 51-60 years old people. There are a few research about unhealthy lifestyle for the recurrence of BPPV.

After performed the quarantine policy to prevent COVID-19 in Hangzhou it was found that the recurrence of BPPV increased significantly than the same period in 2019 (TABLE 1). Therefore, in this retrospective study, we aimed to (1) investigate the risk factors of recurrence for BPPV; and (2) explore the association 
between unhealthy lifestyle and BPPV recurrence. We hypothesized that the recurrence of BPPV is associated with people's unhealthy lifestyle.

\section{Methods}

\subsection{Object of study}

In total, 186 patients were retrospectively enrolled in this study. The patients received the definitive diagnosis and CRM treatment, and finally got cured. All patients were diagnosed BPPV in the Department of Neurology in our hospital. From December 2018 to March, 2020, there were 67 patients who were recurrent (14 males and 53 females) in the observation group, and 119 patients who has no recurrent in the control group in one year follow-up (52 males and 67 females).

\subsection{Diagnostic criteria}

This references the diagnosis standard of BPPV in the 2014 New England Journal (5). All the patients were examined by Dix-Hallpike and roll-tested. Patient's history was required to pinpoint the location of nystagmus. It was required also to diagnose horizontal semicircular canal BPPV and posterior semicircular canal BPPV. Patients who did not complete position test or information was incomplete were excluded.

General information, basic diseases and medication history, time of recumbent position and sleeping, time spent in sunlight, and exercise time were recorded. All patients were follow-up by telephone one year later, some patients came to hospital within one year due to BPPV recurrence. Recurrence of BPPV is defined as the occurrence of two or more times within a year.

Measurement indexes of lifestyle including physical activities, recumbent position time and sun time. Decreased physical activities is mean poor or none exercise lasts for more than two weeks in daily life before BPPV happened. Recumbent position time including decubitus position and sleeping time every day in last two weeks. Poor of sun time is mean none or less than 1 hour everyday lasts for two weeks.

\subsection{Data Analyses}

The measurement data in accordance with normal distribution are expressed by ${ }^{\top} \mathrm{x} \pm \mathrm{S}$, and the comparison between groups is expressed by $t$ test; the numeration data were statistically analyzed with chi-squared test. When $\mathrm{p}<0.05$, the differences between the two groups were deemed to be statistically significant (Table 2). Multivariable logistic regression was performed to identify the recurrence risk factors in all of the patients. All statistical analyses were performed using the STATA statistical software version 15.1 (Table 3).

TABLE 2 Clinical characteristics of no recurrence group and recurrence group in BPPV $(\mathrm{N}=186)$ 
Variables observation group $₫ n=67 \rrbracket$ control group $\llbracket n=119 \rrbracket$ Odds ratio $95 \% \mathrm{Cl} p$-value Total (N) Column (\%) Total (N) Column (\%)

Age, years (mean \pm SD) $50.43 \pm 17.0948 .63 \pm 16.86-46.831-51.7260 .488$ *

Female 5379.106756 .303 .428 1.594-7.746 0.0006\#

MD $34.4843 .361 .3480 .191-8.2230 .701$

Sudden deafness 11.4910 .840 .609

Migraine $45.9797 .560 .7760 .168-2.9210 .683$

Hypertension $68.961915 .970 .5180 .161-1.4480 .178$

Hyperlipemia $1014.932117 .640 .8190 .321-0.9730 .633$

Diabetes 68.96108 .401 .147 0.325-3.692 0.897

CHD $45.9775 .881 .0160 .230-4.1800 .981$

POCI $11.4932 .520 .5860 .011-7.4840 .643$

Mental factors $45.9765 .041 .1960 .239-5.2550 .788$

Mental labor $4364.183630 .254 .1312 .092-8.2000 .000^{\#}$

Poor physical activities 5782.613831 .9312 .15 5.343-29.255 0.000\#

Recumbent position

Time (mean \pm SD) $11.34 \pm 2.309 .02 \pm 2.33-9.484-10.2270 .000$ *\#

Sun time冈1h 4364.185042 .022 .473 1.276-4.823 0.004"

*the P-value was tested by t-test and the others was chi-squared test. \# Gender, prolonged recumbent position time, poor physical activities and sun time, reached statistical significance $(P<0.05)$.

TABLE 3 Multivariate analysis of BPPV patients during 1-year follow-up. 
Variables Odds ratio $95 \%$ confidence interval $p$-value

Age $1.0100 .987-1.0340 .395$

Female $0.3890 .133-1.1410 .086$

Mental labor 2.058 0.499-8.495 0.318

Poor physical activities 5.690 1.650-19.618 0.006*

Recumbent position

Time (mean \pm SD) 1.651 1.370-2.003 0.000*

Sun time $₫ 1 \mathrm{~h} 1.9010 .618-5.8450 .262$

* Poor physical activities and prolonged recumbent position time were significance predictors for the recurrence of BPPV.

\section{Results}

Clinical features and medical history of two group's patients were summarized in Table 2. There was no significant difference was found between the two groups with respect to age, MD, sudden deafness migraine, hypertension, hyperlipemia, diabetes, $C H D, P O C l$, mental factors, and mental labor $(P>0.05)$. But the gender and lifestyle including prolonged recumbent position time and poor physical activities and sun time, reached statistical significance $(P<0.05)$ in patients with recurrence of BPPV compared with controls.

To identify the recurrence predictors of BPPV, multiple binary logistic regression analyses were performed. Due to the strong correlation, recumbent position time and poor physical activities values were found to be independently associated with the recurrence of BPPV in the multiple logistic regression model (Table 3). Every 1 unit increase in prolonged recumbent position time value was shown to cause a 1.651(1.370-2.003) fold increase in predicting the risk of BPPV recurrence (odds ratio, 95\% $\mathrm{Cl}, \mathrm{P}<0.006$ ). Every 1 unit increase in poor physical activities value was shown to cause a 5.69- (1.650-19.618) fold increase in predicting the risk of BPPV recurrence (odds ratio, $95 \% \mathrm{Cl}, \mathrm{P}<0.000$ ).

\section{Discussion}

As we all know, age, gender, sex hormones, osteoporosis, hypertension, hyperlipidemia, diabetes and hyperuricemia are all considered as risk factors of BPPV (6-9). According to previous study that cerebrovascular risk factors influenced BPPV recurrence(10). The morbidity of BPPV in male and female is 1:1.5-2, which is usually high after 40 years old and increases with age (4). In addition, some studies have found that age does not increase the recurrence rate of BPPV (10).We found the recurrence of females was 3.79 fold which significantly higher than male but the female -to-male ratio was 1.82 to 1 in total patients. In theory, with the increase of age, the function of human organs gradually decline. As a 
part of inner ear structure, the metabolism, absorption and regeneration of otoliths are affected, and can easily fall off and leading to the recurrence of BPPV. Previous studies have found that the high morbidity of BPPV in female may be related to widespread osteoporosis(5). It may also be related to the abnormal hormone metabolism in postmenopausal females. But we found there is none correlation with the common BPPV related risk factors in the observation group, reasons for the small number of cases cannot be excluded.

However, our research showed that the recurrence patients of BPPV was very higher than the same period under the quarantine policy in Hangzhou from January 2020 to March 2020. This maybe correlated with their unhealthy lifestyle during COVID-19 spread.

In the life style of BPPV patients in the observation group, the majority of patients has the following characteristics, such as poor physical activities, and prolonged recumbent position time. Especially after implementing the policy on February 4, 2020. It can be seen that prolonged recumbent position time, lack of exercise may be the important pathogenic factors of BPPV recurrence. Van WE had confirmed that $11 \%$ of the dizziness symptoms in Parkinson's patients are likely to have BPPV, which is also considered to be related to exercise reduction (11). It has been suggested that prolonged bed rest may promote calcium carbonate deposition and otolith relaxation in the elliptical capsule (7). The author believes that this viewpoint can better explain the mechanism of the significant increase of BPPV patients in our study. Studies have found that lack of exercise is one of the most important risk factors for BPPV recurrence. Morbidity of female who do not exercise is 2.62 -fold that of regular exercise (12). Regular physical exercise may be a good choice that reduces the risk of BPPV recurrence.

Some studies found that the decrease in the plasma vitamin D level is directly related to the occurrence and recurrence of BPPV $(13,14)$. However, contrary to these finds are other studies which have found that seasonal vitamin D deficiency in winter is not enough to cause BPPV (15). The quarantine policy allowed one person per household family to leave the apartment to undertake supplies shopping once every two days for a period of one hour in Hangzhou. This is for gathering supplies and as such very little skin is exposed to the sunshine, and this may be result in vitamin D deficiency. However, it is regrettable that the plasma vitamin $D$ of these patients had not been determined. Our study also showed that sun time was not associated with BPPV recurrence

The authors suggest that BPPV may not be caused by the falling off of otoliths in the utricle into the semicircular canal. It may be the change of the composition of the endolymph or the deposition of the inherent composition. We proposed the "sediment" theory of BPPV. This kind of deposition is easy to deposit in the posterior or horizontal semicircular canals due to the gravity effect when lying for a long time, but not in the anterior semicircular canals. The deposition of sediments in healthy people may be continuous, which may be absorbed and dissipated due to regular exercise and suitable recumbent position time. For those who lack exercise and prolonged recumbent position time, sediments deposition is affected by gravity, and when they move position such as getting up from a resting position or turning 
over this causes sediment displacement which then results in BPPV. This finding may explain why BPPV occurs several days after trauma, rather than immediately after trauma.

\section{Conclusion}

This study found that the recurrence of BPPV had no obvious relationship with age, gender, migraine, cerebrovascular risk factors, and ear diseases. Poor physical activities and prolonged recumbent position time are important factors to increase the recurrence of BPPV in this study. Changing unhealthy lifestyles may be the solution to decreasing the recurrence of BPPV. The authors speculate that BPPV will probably be a disease that is linked to lack of physical activities.

The limitations of the study was failed to assess the anxiety and depression of all patients.

\section{Abbreviations}

BPPV: Benign paroxysmal positional vertigo; CRM: canalith repositioning maneuvers; SD, standard deviation; MD, Ménière's disease; $\mathrm{CHD}$, coronary heart disease; $\mathrm{POCl}$, posterior circulation cerebral infarction.

\section{Declarations}

\section{Ethics approval and consent to participate}

The study was performed according to the Declaration of Helsinki guidelines, and written informed consent was obtained from all participants. The study protocol was approved by the Tongde Hospital of Zhejiang Province Ethics Committee, and written informed consent was obtained from all patients. The patients in our study only underwent standard treatment without additional interventions for research purposes, so no formal ethics number approval was required.

\section{Consent for publication}

Written informed consent for publication was obtained from all participants.

\section{Competing interests}

The authors declare that they have no competing interests.

Funding: none.

\section{Authors' contributions}

CF conceived the study and design, conducted the experiment, and wrote the manuscript. YL prepared manuscript, and revised this manuscript. ZZ, CJ and SKJ conducted acquisition of subjects and interpretation of data. 


\section{Acknowledgements}

The first author thanks the family for their support, sorry for the children, in order to finish the study without time to accompany their growth. All authors have read and approved the manuscript.

\section{Availability of data and materials:}

The datasets used and/or analysed during the current study are available from the corresponding author on reasonable request.

\section{References}

1. Choi JY, Glasauer S, Kim JH, Zee DS, Kim JS. Characteristics and mechanism of apogeotropic central positional nystagmus. Brain. 2018;141(3):762-775.10.1093/brain/awx381

2. von Brevern M, Radtke A, Lezius F, Feldmann M, Ziese T, Lempert T, et al. Epidemiology of benign paroxysmal positional vertigo: a population based study. J Neurol Neurosurg Psychiatry. 2007;78(7):710-715.10.1136/jnnp.2006.100420

3. Perez P, Franco V, Cuesta P, Aldama P, Alvarez MJ, Mendez JC. Recurrence of benign paroxysmal positional vertigo. Otol Neurotol. 2012;33(3):437-443.10.1097/MAO.0b013e3182487f78

4. Wang YQ, Li JR, Zou SZ, Ding YL. [Clinical features and recurrence rate on benign paroxysmal positional vertigo]. Lin Chung Er Bi Yan Hou Tou Jing Wai Ke Za Zhi. 2019;33(12):11851188.10.13201/j.issn.1001-1781.2019.12.017

5. Kim JS, Zee DS. Clinical practice. Benign paroxysmal positional vertigo. N Engl J Med. 2014;370(12):1138-1147.10.1056/NEJMcp1309481

6. Webster G, Sens PM, Salmito MC, Cavalcante JD, Santos PR, Silva AL, et al. Hyperinsulinemia and hyperglycemia: risk factors for recurrence of benign paroxysmal positional vertigo. Braz J Otorhinolaryngol. 2015;81(4):347-351.10.1016/j.bjorl.2014.09.008

7. Parham K, Kuchel GA. A Geriatric Perspective on Benign Paroxysmal Positional Vertigo. J Am Geriatr Soc. 2016;64(2):378-385.10.1111/jgs.13926

8. Celikbilek A, Gencer ZK, Saydam L, Zararsiz G, Tanik N, Ozkiris M. Serum uric acid levels correlate with benign paroxysmal positional vertigo. Eur J Neurol. 2014;21(1):79-85.10.1111/ene.12248

9. D'Silva LJ, Whitney SL, Santos M, Dai H, Kluding PM. The impact of diabetes on mobility, balance, and recovery after repositioning maneuvers in individuals with benign paroxysmal positional vertigo. J Diabetes Complications. 2017;31(6):976-982.10.1016/j.jdiacomp.2017.03.006

10. Zhu CT, Zhao XQ, Ju Y, Wang Y, Chen MM, Cui Y. Clinical Characteristics and Risk Factors for the Recurrence of Benign Paroxysmal Positional Vertigo. Front Neurol. 2019;10:1190.10.3389/fneur.2019.01190

11. van Wensen E, van Leeuwen RB, van der Zaag-Loonen HJ, Masius-Olthof S, Bloem BR. Benign paroxysmal positional vertigo in Parkinson's disease. Parkinsonism Relat Disord. 2013;19(12):1110- 
1112.10.1016/j.parkreldis.2013.07.024

12. Bazoni JA, Mendes WS, Meneses-Barriviera CL, Melo JJ, Costa Vde S, Teixeira Dde C, et al. Physical activity in the prevention of benign paroxysmal positional vertigo: probable association. Int Arch Otorhinolaryngol. 2014;18(4):387-390.10.1055/s-0034-1384815

13. Ding J, Liu L, Kong WK, Chen XB, Liu X. Serum levels of 25-hydroxy vitamin D correlate with idiopathic benign paroxysmal positional vertigo. Biosci Rep. 2019;39(4).10.1042/BSR20190142

14. Buki $B$, Ecker $M$, Junger $H$, Lundberg $Y W$. Vitamin $D$ deficiency and benign paroxysmal positioning vertigo. Med Hypotheses. 2013;80(2):201-204.10.1016/j.mehy.2012.11.029

15. Parham K, Kuchel GA, McElhaney JE, Haynes L. A Relationship Between Blood Levels of Otolin-1 and Vitamin D. Otol Neurotol. 2018;39(4):e269-e273.10.1097/MAO.0000000000001747

\section{Figures}

\section{Number of BPVV Recurrences}

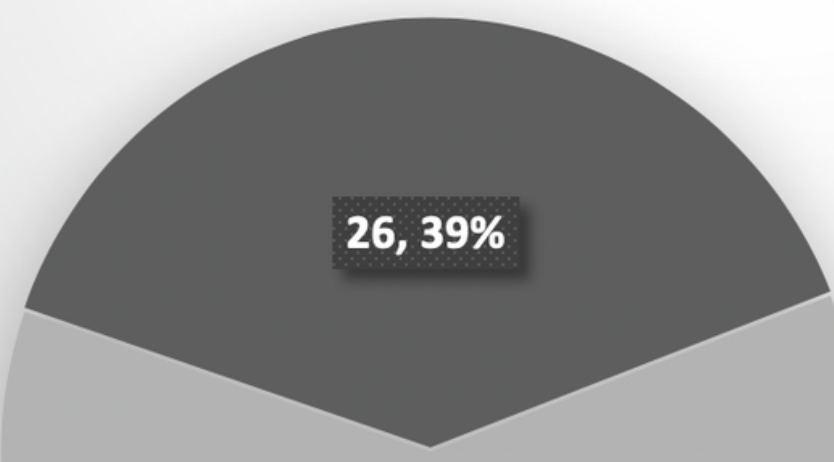

- January 2020 to March 2020

December 2018 - December 2019

$41,61 \%$

\section{Figure 1}

[Table 1] Number of BPVV Recurrences: There were 67 patients with BPPV recurrence from December 2018 to March 2020, and 26 patients (38.8\%) under the quarantine policy from January 2020 to March 2020 . 